\title{
The adaptive immune system in diseases of the central nervous system
}

\author{
David C. Wraith ${ }^{1}$ and Lindsay B. Nicholson ${ }^{1,2}$
}

${ }^{1}$ School of Clinical Sciences and ${ }^{2}$ School of Cellular and Molecular Medicine, University of Bristol, Bristol, United Kingdom.

\begin{abstract}
Tissues of the CNS, such as the brain, optic nerves, and spinal cord, may be affected by a range of insults including genetic, autoimmune, infectious, or neurodegenerative diseases and cancer. The immune system is involved in the pathogenesis of many of these, either by causing tissue damage or alternatively by responding to disease and contributing to repair. It is clearly vital that cells of the immune system patrol the CNS and protect against infection. However, in contrast to other tissues, damage caused by immune pathology in the CNS can be irreparable. The nervous and immune systems have, therefore, coevolved to permit effective immune surveillance while limiting immune pathology. Here we will consider aspects of adaptive immunity in the CNS and the retina, both in the context of protection from infection as well as cancer and autoimmunity, while focusing on immune responses that compromise health and lead to significant morbidity.
\end{abstract}

\section{Immune privilege}

A range of mechanisms exist to limit immune responses in the CNS; indeed, the CNS is considered to be an immune-privileged site. As early as 1921, it was shown that rat sarcoma cells grow well in mouse brain parenchyma but not when transplanted under the skin or into muscle (1). For decades thereafter, it was assumed that the CNS and retina enjoy immune privilege because they are hidden behind the blood-brain barrier (BBB), blood-cerebrospinal fluid barrier (BCSFB), or the blood-retinal barrier (BRB) (Figure 1). However, the view that the CNS is completely ignored by the immune system has turned out to be overly simplistic. This is in part because immune privilege is relative rather than absolute; the immune response to nontumor foreign tissue in the CNS is delayed rather than prevented (2). This delay is related to several factors - the CNS lacks conventional lymphoid drainage (3) and CNS-derived antigen may be transported to cervical lymph nodes in the fluid phase (4) or associated with DCs following tissue trauma (5). The parenchyma of the normal brain and spinal cord has a limited capacity for antigen processing and presentation, since it contains few professional APCs and neurons only express MHC under exceptional conditions (6). The efferent arm of the immune response is also hindered, since lymphocytes have to be activated before they can cross the $\operatorname{BBB}$ or $\operatorname{BRB}(7,8)$, and even then this transmigration process is challenging. Once in CNS tissue, the environment remains inherently hostile to activated lymphocytes expressing FAS, ligation of which by FAS ligand (FASL), expressed on all cells in the CNS, results in death by apoptosis (9, 10). Microglia, the innate immune cells of the CNS, further respond to inflammation by upregulation of immunoregulatory molecules including B7-H1 (11) and IDO (12), while neurons protect themselves by secreting TGF- $\beta$ upon contact with activated lymphocytes (13). FAS and TGF- $\beta$ have also been implicated in the suppression of immune responses in the eye (refs. 14, 15, and Figure 2).

\section{Immune surveillance}

The nature and origin of APCs in the CNS is only now becoming clear. Resident brain microglial cells are derived from primi-

Conflict of interest: David C. Wraith has a financial interest in Apitope, which is developing antigen-specific therapies for autoimmune diseases.

Citation for this article: J Clin Invest. 2012;122(4):1172-1179. doi:10.1172/JCI58648. tive myeloid progenitors that differentiate in the yolk sac (16), although bone marrow-derived cells may reconstitute the CNS following trauma (17). Greter and colleagues have shown that immune responses in the CNS depend on CD11 $\mathrm{c}^{+}$cells found in the juxtavascular parenchyma, with cell processes extending into the glia limitans (18). Importantly, these cells may be blood born or alternatively derive from an intraparenchymal, microglial precursor stimulated with GM-CSF (19). Cells sharing the properties of conventional DCs have recently been found in the meninges and choroid plexus of healthy mouse brain (20). These cells are derived from bone marrow pre-DC progenitors and share morphological characteristics, gene expression patterns, and the ability to present antigen with splenic DCs (20). Apart from these populations of DCs, the CNS parenchyma is relatively devoid of APCs. This all changes, however, in the inflamed CNS or retina when myeloid $\left(\mathrm{CD} 11 \mathrm{~b}^{+}\right)$DCs flood into the site, amplify the immune response, and promote epitope spreading (21-23).

The non-inflamed brain and retina are protected by vascular endothelium at the BBB and BRB, while epithelial cells of the choroid plexus form the BCSFB (Figure 3). Furthermore, astrocytic end feet and the parenchymal basement membrane form a further barrier, the glia limitans. Nevertheless, CSF from individuals with no inflammatory neurological disease contains about 150,000 $\mathrm{T}$ lymphocytes (24). These cells circulate through the CSF for approximately six hours before returning to the circulation (24) - a low rate of cell traffic when compared with peripheral lymphocyte recirculation (25). The $\mathrm{T}$ cells in human CSF are mainly effector memory (CD45RA ${ }^{-}, \mathrm{CD}_{27}{ }^{+}, \mathrm{L}^{-}$selectin $^{\text {hi }}$ ), and the majority are CD4 positive (26). This phenotype permits trafficking through extralymphoid tissue as well as subsequent return to the lymphatic system via high-endothelial venules. Activated lymphocytes make formal contact with the BBB via $\alpha 4$-integrin and endothelial VCAM-1 (27) and cross the barrier by diapedesis. This is a difficult process, especially in the non-inflamed CNS, although entry to the leptomeningeal compartment can occur more readily in a P-selectindependent manner (28). Even then, entry to the CNS parenchyma is dependent on further encounter with cognate antigen. If antigen is seen, then the immune cells mount an inflammatory response, draw other immune cells into the specific site, and then collectively breech the glia limitans to infiltrate the parenchyma. 


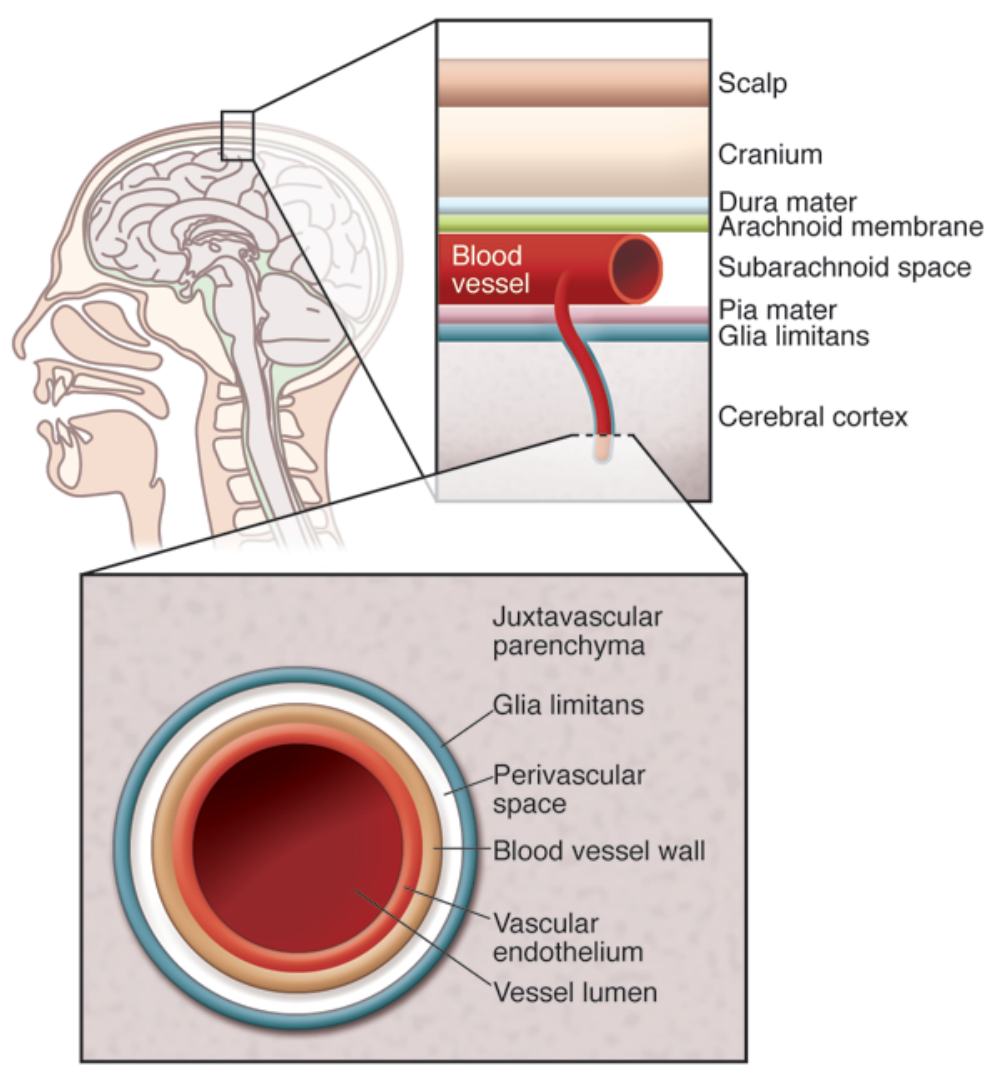

\section{Figure 1}

Physical barriers protecting the brain. The brain is the most highly protected organ in the body. It is protected from physical insult by the skull and associated tissues. The BBB provides protection from pathogens in the blood and from the cells and antibodies of the immune system. The BBB is more effective than other vascular-tissue barriers because the endothelial cells form tight junctions of high electrical resistance that limit transcellular movement of molecules. The glia limitans, formed from parenchymal basement membrane and astrocytic foot processes, forms a further barrier between blood and neuronal tissue.
Lymphocyte migration into and within the CNS is regulated by chemokines and their receptors. Without concomitant inflammation, $\mathrm{CD}^{+}$migration outside of blood vessels is constrained to pathways that run along their axes (29) and is different from the random motility of $\mathrm{CD}^{+}$cells $(29-31)$. This confinement is regulated by the interaction of the chemoattractant CXCL12 with the receptor CXCR4, expressed on the surface of lymphocytes. The migration of leukocytes into the CNS may be modulated by sequestration of CXCL12 by other receptors (32), or by the physical redistribution of CXCL12 that occurs in MS and the disease model EAE $(30,31)$. In addition, blockade of CXCR4 allows CD4 T cells to escape from their perivascular containment and penetrate deeper into brain parenchyma $(29,30)$. Collectively these mechanisms ensure that immunosurveillance within the normal CNS occurs at a slower pace than in the periphery and is biased to recently activated $\mathrm{CD} 4^{+}$cells with a phenotype that allows them to traffic back to secondary lymphoid tissue once they leave the nervous system. However, immunosurveillance of the CNS is a critical mechanism, as illustrated recently by the observation of complications associated with antibody therapies for MS that block this process. Treatment with natalizumab, an anti- $\alpha 4$-integrin, increases the risk of progressive multifocal leukoencephalopathy (PML) caused by the John Cunningham (JC) Virus (33). This is virtually never seen in immune-competent individuals (34), attesting to the effectiveness of the immune surveillance of CNS tissue.

\section{CNS disease associated with infection}

Infections of the CNS frequently cause devastating disease with long-term neurological sequelae. It is also appreciated that the tissue damage caused by such fulminant inflammatory responses in the brain may have more severe consequences than the infection itself. However, life-threatening inflammation is not the only manifestation of immune responses within the CNS, and certain viral infections of the CNS and meninges can produce transient symptoms that resolve completely. Studying adaptive immune responses within immunoprivileged sites that can resolve without clinical sequelae is challenging, especially in humans. In the retina, such events manifest as multiple evanescent white dot syndrome, putative immune granulomas often of unknown cause that have a spectrum of clinical outcomes from resolution to chronic disease (35). Other examples of clinically silent pathology include aseptic meningitis, most commonly caused by enteroviruses, in which the infection is cleared from the tissue, and infection followed by latency as caused by herpes viruses. In this latter case, immunosuppression can promote reactivation of the virus - for example, in patients receiving treatment with antibodies that deplete peripheral lymphocytes (36), which can lead to acute retinal pathology (37). The precise origin of the reactivation is difficult to determine, but in animal models, latent virus can be detected in the trigeminal ganglion and hypothalamus (38). Together this evidence demonstrates that the CNS can support effective adaptive immune responses while preserving normal function.

Infectious agents may cause neurological disease by a direct lytic effect, exemplified by Venezuelan equine encephalitis virus in children and West Nile virus in older adults (39). Alternatively, damage can be caused by the immune response to the virusinfected cells; examples of viruses causing immunopathology in the CNS include Eastern equine encephalitis virus in humans and Sindbis virus in mice (40). Human T-lymphotrophic virus type 1 
A

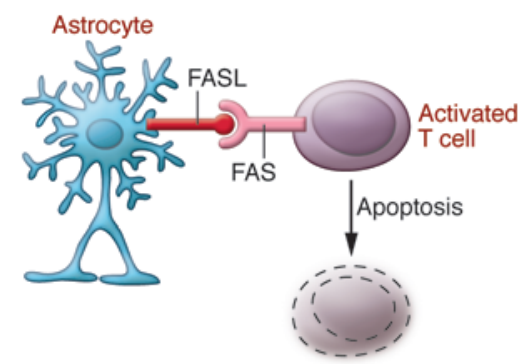

B

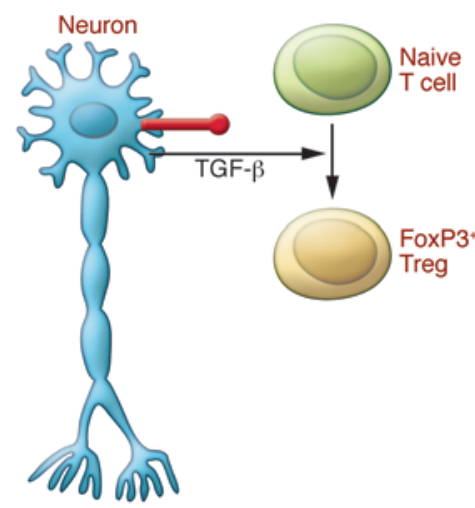

C

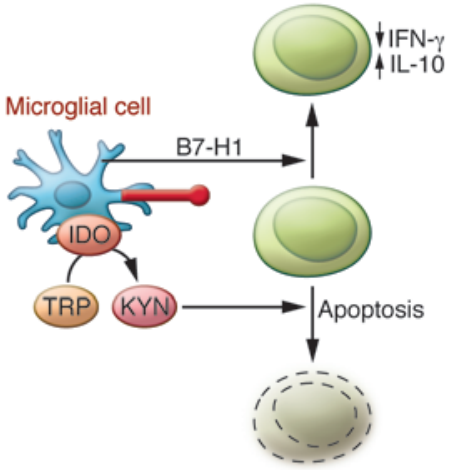

Figure 2

Immunological barriers protecting the brain. Cells of the CNS, including (A) astrocytes, (B) neurons, and (C) microglial cells express FASL. Activated T cells upregulate FAS and are, therefore, susceptible to apoptosis in the CNS. (B) Neurons respond to contact with T cells by secreting TGF- $\beta$, which in turn promotes the generation of regulatory T cells. (C) Resident microglial cells express B7-H1, a costimulatory molecule that promotes secretion of the anti-inflammatory cytokine IL-10, and indoleamine 2,3-dioxygenase (IDO), which converts tryptophan (TRP) to kynurenic acid (KYN). Metabolites of tryptophan induce FAS-independent apoptosis in neighboring cells.

(HTLV-1) infection may result in HTLV-associated myelopathy (HAM), also known as tropical spastic paraparesis (TSP), but this must occur by a different, indirect mechanism. HTLV-1 infects both CD4 and CD8 lymphocytes and causes T cell leukemia/ lymphoma among CD4 cells (41). Interestingly, however, there is little or no HTLV-1 infection of CNS cells in HAM/TSP (42), and risk of developing HAM/TSP correlates most strongly with the proportion of peripheral blood mononuclear cells carrying integrated HTLV-1 provirus. In contrast, a strong CD8 antiviral response reduces proviral load and hence reduces the risk of HAM/TSP (43). A poor CD8 response to the virus results in a high number of infected and activated CD4 T cells, and a high percentage of these cells is able to enter the CNS, where they form an immune battleground between the infected cells and HTLV-1-specific lymphocytes. The latter secrete inflammatory cytokines and metalloproteinases, and these cause tissue damage in a bystander fashion.

A further mechanism of neuronal disease associated with infection is revealed through studies of Sydenham chorea. This disorder may occur months after group A streptococcal infection and is associated with antibodies against basal ganglia (44). Monoclonal antibodies from patients with Sydenham chorea were shown to react with intracellular tubulin, extracellular lysoganglioside GM1, and the GlcNAc epitope of streptococcal group A carbohydrate, consistent with a mechanism of molecular mimicry (45). Antibodies from Sydenham chorea patients react strongly with cytoplasmic antigens in human caudate and subthalamic nuclei as well as cerebral cortex neurons (44). These antibodies are specific to patients with symptoms of chorea and are not seen in rheumatic heart disease, and their levels correlate with severity and duration of choreic symptoms. Whether or not anti-tubulin or anti-lysoganglioside antibodies cause the pathology remains unclear. Some antibodies against lysoganglioside have, however, been shown to activate calcium/calmodulin-dependent protein kinase II in human neuronal cells (46). One question that arises is how such cross-reactive antibodies cross the BBB or BCSFB in the first place. Evidence from a study of systemic lupus erythematosus (SLE) has shown that antidsDNA antibodies can cross-react with NMDA receptor on the cell surface and induce apoptosis of neurons in the hippocampus and lateral amygdala (47). Importantly, in this study Huerta and colleagues showed that the BBB had first to be disrupted for these antibodies to cause pathology. The phenomena of molecular mimicry and the induction of cross-reactive antibodies are probably more common than we appreciate. The integrity of the $\mathrm{BBB}$ and BCSFB means, however, that this rarely manifests as a clinical feature of either the infection or autoimmune condition. In summary, infectious agents may cause neurological disease through a direct lytic effect, by inducing immunopathology directed against CNS tissue, by induction of immune responses that damage CNS tissue in a bystander fashion, or through induction of molecular mimicry.

The control of virus infection and survival of the host, especially in response to viruses adapted to evade the immune system, may require the production of antibodies within the CNS and/or the generation of protective but non-lytic effector functions. For example, coronavirus infection of mice results in acute encephalomyelitis followed by persistent infection (48). Serum antibody levels correlate with levels of tissue antibody-secreting cells in the periphery and decline following viral clearance. However, antibody-secreting cells persist in the CNS consistent with intrathecal antibody synthesis. The CNS is therefore able to support antibody-secreting cells even after resolution of virus infection. Evidence for long-term persistence of $\mathrm{CD}_{103^{+}}$pathogen-specific memory $\mathrm{CD}^{+}$cells in the $\mathrm{CNS}$ comes from elegant work using vesicular stomatitis virus (49). Furthermore, human tissue studies have identified $\mathrm{CD}^{+}$cells associated with trigeminal ganglia infected with herpes simplex virus (50). Although granzyme B was expressed by these cells, they appeared to be non-cytolytic. Similar findings have been described in animal models of herpesvirus infection, in which both granzyme- and cytokine-dependent effector mechanisms are important for 
A

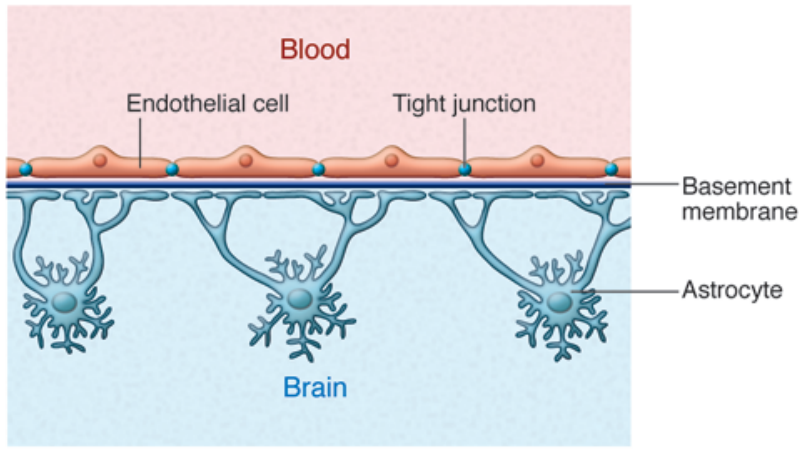

B

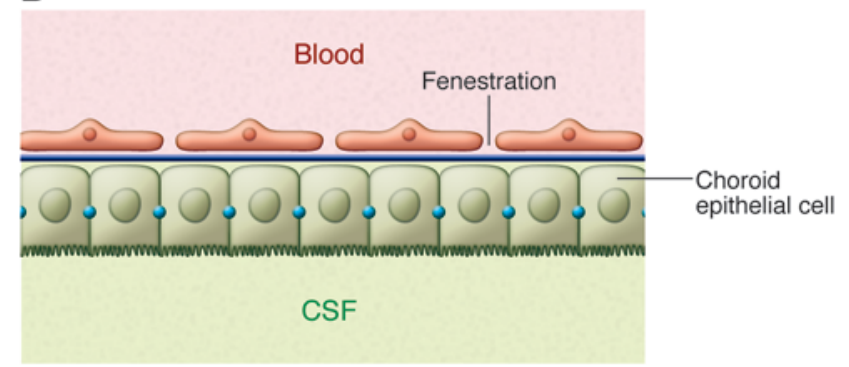

Figure 3

Comparison of the BBB and BCSFB. Brain barriers are made up of the endothelial BBB and the epithelial BCSFB. (A) The BBB has two protective layers, the endothelium with tight junctions and the glia limitans, made up of parenchymal basement membrane and astrocyte foot processes. (B) In contrast to the CNS parenchyma, microvessels in the choroid plexus are fenestrated, allowing free diffusion between the blood and CSF. The BCSFB is made up of the choroid epithelial cells with their unique tight junctions.

maintaining the latent state of the virus (51). In this latter model, it was possible to demonstrate lytic granule-dependent degradation of viral protein without concomitant neuronal cell death. Taken together, these studies suggest that the immune system can control CNS infections using mechanisms that limit tissue damage.

\section{Paraneoplastic diseases of the CNS}

In addition to the movement disorders associated with infection by group A streptococcus or autoimmunity (e.g., SLE), an important subset of paraneoplastic neurological diseases (PNDs) is linked to cancer (52). These conditions are often associated with lymphocyte pleocytosis in the CSF and appearance of oligoclonal bands (multiple bands of immunoglobulin detected by electrophoresis, evidence of B cell clonal expansion) and, more specifically, anti-neuronal antibodies. Tumors involved in PND may express neuroendocrine proteins (e.g., small-cell lung cancer [SCLC] and neuroblastoma), affect organs involved in immune regulation (thymoma), or contain mature or immature neuronal tissue (teratomas) (Table 1).

In some cases, the pathogenic role of paraneoplastic antibodies is clear. For example, antibodies directed to the NR1 subunit of the NMDA receptor cause its internalization and the associated reduction in receptor signaling (53). Furthermore, stiff person syndrome is unequivocally linked to antibodies that interfere with the GABA-receptor pathway (GAD65, amphiphysin, GABARAP); as yet, however, which of these antibodies results in pathology is less clear (52). The same is true for antibodies associated with other movement disorders, especially those specific for cytoplasmic antigens, in which the pathogenic link remains obscure. In many cases, paraneoplastic movement disorders are successfully treated by removal of the tumor with or without additional immune suppression.

\section{Autoimmune diseases of the CNS}

Autoimmune diseases are characterized by strong association with genes, usually class II, in the MHC (54); weak association with a range of other genes encoding proteins known to regulate immune responses (55); lymphocyte infiltration of the affected tissue (56); raised levels of class-switched autoantibodies in serum or the affected tissue (57); higher frequency of activated or memory lymphocytes (CD45RO ${ }^{+} \mathrm{T}$ cells in humans) specific for self-antigens (58); and the suppression of disease progression through treatment with strong immune-suppressive drugs (59). Autoimmune diseases that affect the CNS include MS and neuromyelitis optica (NMO).

Until recently, NMO, or Devic's disease, was considered to be a form of MS, since the immune system targets myelin in both diseases (60). Pathogenic lesions in MS are found in both brain and spinal cord, whereas the autoimmune attack in NMO specifically affects the spinal cord and optic nerve; hence, the symptoms of NMO are loss of vision and spinal cord function. Definite diagnosis of NMO is based on evidence of optic neuritis, acute myelitis, and at least 2 of the following additional criteria: (a) contiguous spinal cord MRI lesions over 3 vertebral segments, (b) brain MRI not meeting criteria for MS, and (c) presence of antibodies against aquaporin-4, a highly specific marker of NMO (61). These antibodies are detectable in at least $80 \%$ of patients with NMO and have been shown to cause relevant pathology when either transferred directly into the CNS of mice (62) or injected into animals with EAE that have a disrupted BBB (63), allowing free passage of IgG antibodies into the brain. There is also evidence for increased T-cell immunity against aquaporin-4 in NMO (64). Importantly, MS is more common in populations of mixed European descent, whereas $\mathrm{NMO}$ is relatively more frequent in individuals not of mixed European descent, implying a strong genetic contribution to susceptibility. In MS there is a clear association with the HLA-DRB1*1501 allele in populations of European origin, while HLA-DRB1*0301 and DRB $1 * 0405$ are more common in Sardinia (65); these alleles of the MHC class II HLA-DR locus constitute the strongest genetic association with MS (66). Similarly, NMO is associated with HLA class II, and it appears, from the few small studies conducted, that the associated allele depends on ethnicity. That is, NMO has been associated with HLA-DP*0501 in Japan (67), whereas patients in France (68) and Brazil (69) expressing the anti-aquaporin antibody are likely to be HLA-DRB ${ }^{*} 03$. Therefore, $\mathrm{NMO}$ is an autoimmune disease of the CNS that shares some of the features with MS but is distinct both genetically and in immunopathology.

Narcolepsy fulfils some, but not all, of the features of an autoimmune disease. This disabling CNS disorder causes daytime sleepiness and sleep attacks that develop because of a deficiency of hypocretin-producing neurons in the hypothalamus (70). The majority of patients with narcolepsy carry the HLA DQB ${ }^{*} 0602$ allele (71). Recent studies have also revealed disease-associated polymorphisms in genes encoding the TCR $\alpha$-chain (72), TNF- $\alpha$ (73) 


\section{Table 1}

Examples of paraneoplastic movement disorders

\begin{tabular}{llll}
\hline Disease & Related tumor & Antibody target & Reference \\
Chorea & SCLC and thymoma & CRMP-5 & 94 \\
NMDA receptor encephalitis & Teratoma (in women) & NMDAR (NR1) & 95 \\
Brainstem encephalitis & Testis (in men under 50 years of age), & MA2 & 96 \\
& solid tumor (in men over 50), & & \\
& lymphoma (in women) & Yo & 97 \\
Cerebellar degeneration & Breast, ovary & TR & 98 \\
& Hodgkin's lymphoma & VGCC & 99 \\
Stiff person syndrome & SCLC & GAD65, amphiphysin, GABARAP \\
Opsoclonus-myoclonus & SCLC, breast & Not known & 100,101 \\
& Neuroblastoma (in children); & & 102 \\
\hline
\end{tabular}

and the TNF receptor II (74). Despite such tantalizing immunogenetic evidence, there is insufficient definitive proof that narcolepsy is an autoimmune condition. Oligoclonal bands are not seen in the CSF (75), nor are there signs of inflammation in the hypothalamus in postmortem studies $(76,77)$. As discussed above, neurological disturbance can result from various mechanisms, ranging from direct immune attack, bystander activation, or molecular mimicry. It remains possible that a subtle, local bystander activation mechanism, distinct from the type of pathology seen in MS and NMO, could account for the specific depletion of hypothalamic neurons producing hypocretin peptides, but this awaits further investigation.

The available therapies for many autoimmune diseases are not curative and are often associated with unacceptable side effects. Over the past 20 years, advances in our understanding of the immune pathology of autoimmune diseases have led to new treatments that offer significant advantages over previous therapies. This is well illustrated in the case of MS, for which various treatment approaches have recently entered clinical trials or have been added to the armamentarium of the neurologist (Figure 4). Interferon- $\beta$ was introduced in the 1980s, in the belief that MS was caused by an unknown virus, and was shown to suppress inflam- mation in some but not all patients. Recently, Axtell and colleagues provided insight into why the effects of interferon- $\beta$ may vary (78). In the mouse EAE model, interferon- $\beta$ was shown to suppress disease caused by myelin-specific Th1 cells but to exacerbate Th17associated disease. Interestingly, patients who do not respond to treatment with interferon- $\beta$ have constitutively high levels of IL-17F (78). Extrapolating these findings to the general population of patients with MS, one could speculate that the efficacy of interferon- $\beta$ in a given individual might depend on the balance of Th1 and Th17 cells causing pathology. Relative cytokine production by peripheral blood cells in response to myelin antigens could be used to stratify patients and thereby improve the effective treatment of MS with interferon- $\beta$.

There is now no doubt that MS is an autoimmune disease associated with the adaptive immune response to myelin antigens. Most strikingly, patients with relapsing MS have shown an impressive reduction in disability at 6 months after treatment with the CD52targeting monoclonal antibody alemtuzumab (Campath $1 \mathrm{H})(79)$. This antibody has a profound and long-lasting effect on the CD4 lymphocyte count, and this alone implicates CD4 T cells as key in controlling the pathogenesis of MS. Treatment with rituximab, an antibody targeting the CD20 molecule on B cells and leading to

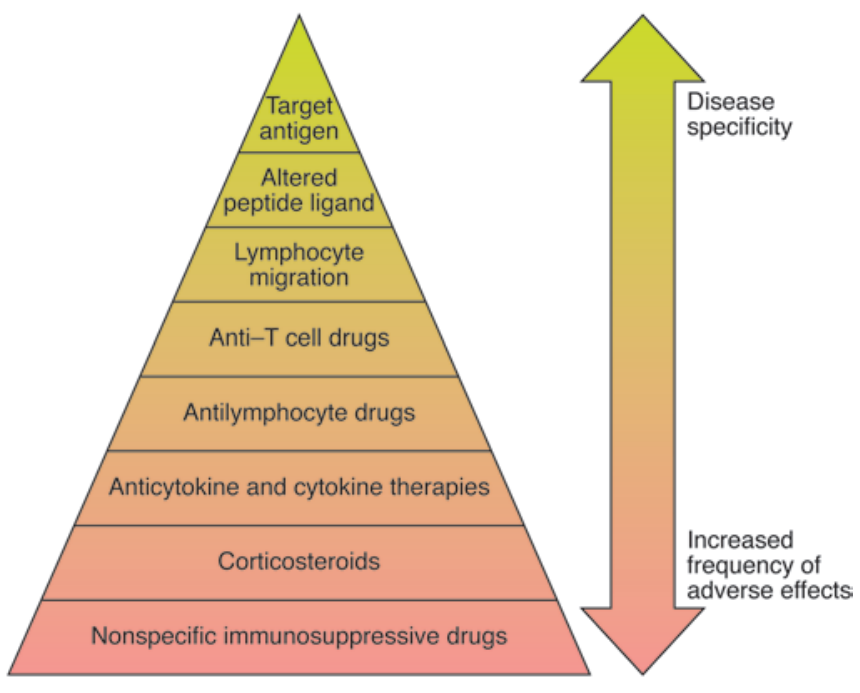

\section{Figure 4}

Current and future therapies for MS. The holy grail for treatment of autoimmune disease is to design a drug that will selectively target the cells causing the disease, avoid nonspecific immune suppression, and have minimal adverse effects. Increasingly specific approaches target the adaptive immune response to antigens in the CNS. Examples of these include antilymphocyte drugs (alemtuzumab), anti-T cell drugs (daclizumab), drugs targeting lymphocyte migration (natalizumab and fingolimod), APL (GA), and the target antigen (myelin peptides or DNA vaccine). 


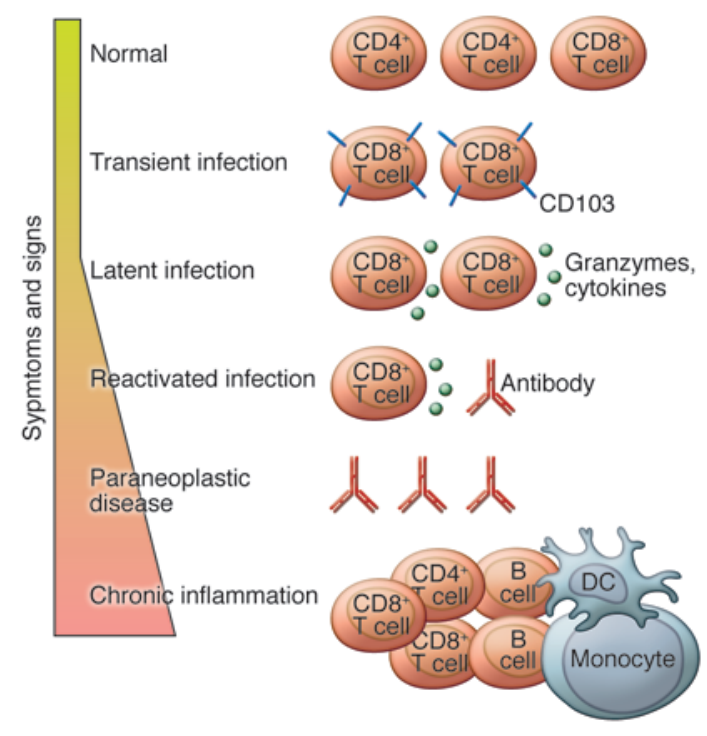

B cell depletion, has also been shown to lower the burden of brain lesions in MS and significantly reduce relapses (80). This may suggest a previously underappreciated role for autoantibodies in immune pathology, or alternatively an important role for B cells in the presentation of antigen to pathogenic CD4 T cells. Work in the EAE model previously revealed the role of VLA-4 as the integrin involved in lymphocyte traffic into the CNS; the anti-VLA-4 antibody HP2/1 prevented onset of EAE in the Lewis rat model (27). Natalizumab, the human equivalent of HP2/1, has been highly effective in reducing inflammatory lesions in MS. Clinical trials have, however, revealed an increased susceptibility to PML in patients treated with natalizumab (81). PML is caused by the reactivation of the JC Virus polyomavirus in the CNS of immune-compromised individuals (82). Similar viral complications may also arise upon treatment with fingolimod (Gilenya), a sphingosine-1 phosphate (S1P) receptor modulator. S1P signaling is required for lymphocytes to egress from lymph nodes. Hence, treatment with fingolimod prevents lymphocytes leaving the lymph nodes and entering the CNS. In a recent trial, fingolimod significantly lowered relapse rate in relapsing-remitting MS (83). However, two fatal viral infections, disseminated varicella and herpes simplex encephalitis, occurred among 369 patients treated at the higher dose of the drug (83). Clearly, further analysis of dose and duration of treatment will be required to assess the long-term safety of drugs that modulate lymphocyte migration.

An antigen-specific "tolerogenic" approach specifically targeting the TCRs of pathogenic cells is considered the holy grail for immunotherapy (84). Altered peptide ligands (APLs) are variants of $\mathrm{T}$ cell epitopes designed to alter the response of $\mathrm{T}$ cells by inducing apoptosis, anergy, or modulation of cytokine secretion (85). The prototypic APL for treatment of autoimmune disease is copolymer 1 , also known as copaxone or glatiramer acetate (GA) (86). GA is thought to work by modulating myelin basic protein-specific (MBP-specific) T cells toward a Th2/anti-inflammatory phenotype (87) or, alternatively, by upregulating IL-10 secretion by CD8 cells (88). APL more specifically targeting the TCRs of myelin-specific cells have been designed and tested in clinical trials. High doses of APL, based on MBP peptide 83-99, caused allergic complications when injected subcutaneously (89) and may have led to dis-

\section{Figure 5}

Adaptive immune responses in the CNS. Immune responses in the CNS may be helpful or harmful. Constant immune surveillance is required to control infections in the CNS, to control transient infections, or to maintain latent infections. Depletion of immune cells may lead to virus reactivation, while cross-reacting antibodies, which arise as a result of infection, autoimmune disease, or cancer, may cause movement disorders. Chronic inflammation arises when the adaptive immune response fails to eradicate an infection or alternatively responds to a CNS antigen, leading to autoimmune disease. ease exacerbation in some patients (90). It was previously shown that an antagonist for one TCR may function as an agonist for the next (91); this impugns the advantage of using APL over the native sequence. Since the early studies with APL, antigen-specific therapies based on peptides and DNA vaccines have been developed. High-dose treatment with MBP peptide 82-98 led to a reduction in anti-MBP antibodies and delayed disease progression in the HLA-DR2/DR4 subgroup of patients (92). A DNA vaccine encoding MBP, with $\mathrm{CPG}$ motifs replaced by $\mathrm{GPG}$, was recently tested in MS patients (93). Treatment with a $0.5-\mathrm{mg}$ dose of DNA resulted in the reduction of new lesions in the CNS, coinciding with a decrease in the Th1 response to myelin antigens. Whether antigenspecific therapy will be sufficiently powerful to reduce progression of disease cannot be determined without further testing. One can envision a situation in which disease is treated by short-term dosage with a strong, nonspecific immune suppressant, with disease suppression being maintained in the long term with a safer, antigen-specific approach.

\section{Conclusion}

In summary, the CNS has co-evolved a close relationship with the immune system that allows a sophisticated and nuanced manifestation of the normal inflammatory process (Figure 5). An ongoing immune response within the immune-privileged tissue modifies its immunosurveillance, and this is characterized by changes in the immune cell content of the target organ. Such changes can be successful in controlling local pathology while preserving function but may alter the risk of CNS sequelae following peripheral immune activation.

\section{Acknowledgments}

D.C. Wraith acknowledges financial support from the Wellcome Trust \& MS Society UK. L.B. Nicholson acknowledges support from the National Eye Research Centre \& MS Society UK.

Address correspondence to: David C. Wraith, Department of Pathology and Microbiology, University of Bristol, University Walk, Bristol BS8 1TD, United Kingdom. Phone: 44.117.928.7883; Fax: 44.117.928.7896; E-mail: D.C.Wraith@bristol.ac.uk. 
1. Shirai Y. On the transplantation of the rat sarcoma in adult heterogeneous animals. Jap Med World. 1921;1:14-15.

2. Galea I, Bechmann I, Perry VH. What is immune privilege (not)? Trends Immunol. 2007;28(1):12-18.

3. Weller RO, Galea I, Carare RO, Minagar A. Pathophysiology of the lymphatic drainage of the central nervous system: Implications for pathogenesis and therapy of multiple sclerosis. Pathophysiology. 2010;17(4):295-306.

4. Weller RO, Engelhardt B, Phillips MJ. Lymphocyte targeting of the central nervous system: a review of afferent and efferent CNS-immune pathways. Brain Pathol. 1996;6(3):275-288

5. Karman J, Ling C, Sandor M, Fabry Z. Initiation of immune responses in brain is promoted by local dendritic cells. J Immunol. 2004;173(4):2353-2361.

6. Neumann H, Cavalie A, Jenne DE, Wekerle H. Induction of MHC class I genes in neurons. Science. 1995;269(5223):549-552.

7. Wekerle H, Linington C, Lassmann $\mathrm{H}$, Meyermann R. Cellular immune reactivity within the CNS Trends Neurosci. 1986;9:271-277.

8. Prendergast RA, et al. T cell traffic and the inflammatory response in experimental autoimmune uveoretinitis. Invest Ophthalmol Vis Sci. 1998 39(5):754-762.

9. Bechmann I, Mor G, Nilsen J, Eliza M, Nitsch R, Naftolin F. FasL (CD95L, Apo1L) is expressed in the normal rat and human brain: evidence for the existence of an immunological brain barrier. Glia. 1999;27(1):62-74.

10. Flugel A, et al. Neuronal FasL induces cell death of encephalitogenic T lymphocytes. Brain Pathol. 2000;10(3):353-364.

11. Magnus T, et al. Microglial expression of the B7 family member B7 homolog 1 confers strong immune inhibition: implications for immune responses and autoimmunity in the CNS. J Neurosci. 2005;25(10):2537-2546.

12. Kwidzinski E, et al. Indolamine 2,3-dioxygenase is expressed in the CNS and down-regulates autoimmune inflammation. Faseb J. 2005; 19(10):1347-1349

13. Liu Y, Teige I, Birnir B, Issazadeh-Navikas S. Neuron-mediated generation of regulatory $\mathrm{T}$ cells from encephalitogenic T cells suppresses EAE. Nat Med. 2006;12(5):518-525.

14. Griffith TS, Yu XH, Herndon JM, Green DR, Ferguson TA. CD95-induced apoptosis of lymphocytes in an immune provileged site induces immunological tolerance. Immunity. 1996;5(1):7-16.

15. Williams GA, Mammolenti MM, Streilein JW. Studies on the induction of anterior chamber-associated immune deviation (ACAID). III Induction of ACAID depends upon intraocular transforming growth factor-beta. Eur J Immunol. 1992;22(1):165-173.

16. Ginhoux F, et al. Fate mapping analysis reveals that adult microglia derive from primitive macrophages. Science. 2010;330(6005):841-845.

17. Hickey WF. Migration of hematogenous cells through the blood-brain barrier and the initiation of CNS inflammation. Brain Pathol. 1991;1(2):97-105.

18. Greter M, et al. Dendritic cells permit immune invasion of the CNS in an animal model of multiple sclerosis. Nat Med. 2005;11(3):328-334.

19. Prodinger C, et al. CD11c-expressing cells reside in the juxtavascular parenchyma and extend processes into the glia limitans of the mouse nervous system. Acta Neuropathol. 2011;121(4):445-458.

20. Anandasabapathy N, et al. Flt3 L controls the development of radiosensitive dendritic cells in the meninges and choroid plexus of the steady-state mouse brain. J Exp Med. 2011;208(8):1695-1705.

21. Lehman PV, Forsthuber T, Miller A, Sercarz EE. Spreading of T-cell autoimmunity to cryptic determinants of an autoantigen. Nature. 1992 358(6382):155-157.
22. Bailey SL, Schreiner B, McMahon EJ, Miller SD. CNS myeloid DCs presenting endogenous myelin peptides 'preferentially' polarize CD4+ T(H)-17 cells in relapsing EAE. Nat Immunol. 2007;8(2):172-180.

23. Kerr EC, Raveney BJ, Copland DA, Dick AD, Nicholson LB. Analysis of retinal cellular infiltrate in experimental autoimmune uveoretinitis reveals multiple regulatory cell populations. J Autoimmun. 2008;31(4):354-361.

24. Engelhardt B, Ransohoff RM. The ins and outs of T-lymphocyte trafficking to the CNS: anatomical sites and molecular mechanisms. Trends Immunol. 2005;26(9):485-495.

25 . Young AJ. The physiology of lymphocyte migration through the single lymph node in vivo. Semin Immunol. 1999;11(2):73-83.

26. Kivisakk P, et al. Human cerebrospinal fluid central memory CD4+ T cells: evidence for trafficking through choroid plexus and meninges via P-selectin. Proc Natl Acad Sci U S A. 2003;100(14):8389-8394.

27. Yednock TA, Cannon C, Fritz LC, Sanchez-Madrid F, Steinman L, Karin N. Prevention of experimental autoimmune encephalomyelitis by antibodies against alpha 4 beta 1 integrin. Nature. 1992; 356(6364):63-66

28. Carrithers MD, Visintin I, Kang SJ, Janeway CA Jr. Differential adhesion molecule requirements for immune surveillance and inflammatory recruitment. Brain. 2000;123(pt 6):1092-1101.

29. Siffrin V, et al. Differential immune cell dynamics in the CNS cause CD4+ T cell compartmentalization. Brain. 2009;132(pt 5):1247-1258.

30. McCandless EE, Wang Q, Woerner BM, Harper JM, Klein RS. CXCL12 limits inflammation by localizing mononuclear infiltrates to the perivascular space during experimental autoimmune encephalomyelitis. J Immunol. 2006;177(11):8053-8064.

31. McCandless EE, Zhang B, Diamond MS, Klein RS. CXCR4 antagonism increases $\mathrm{T}$ cell trafficking in the central nervous system and improves survival from West Nile virus encephalitis. Proc Natl Acad Sci US A. 2008;105(32):11270-11275

32. Cruz-Orengo L, et al. CXCR7 influences leukocyte entry into the CNS parenchyma by controlling abluminal CXCL12 abundance during autoimmunity. J Exp Med. 2011;208(2):327-339.

33. Kappos L, et al. Natalizumab treatment for multiple sclerosis: updated recommendations for patient selection and monitoring. Lancet Neurol. 2011;10(8):745-758.

34. Weber T. Progressive multifocal leukoencephalopathy. Neurol Clin. 2008;26(3):833-854.

35. Ben Ezra D, Forrester JV. Fundal white dots: the spectrum of a similar pathological process. $\mathrm{BrJ}$ Ophthalmol. 1995;79(9):856-860.

36. Keating MJ, et al. Therapeutic role of alemtuzumab (Campath-1H) in patients who have failed fludarabine: results of a large international study. Blood. 2002;99(10):3554-3561

37. Moon SJ, Mieler WF. Retinal complications of bone marrow and solid organ transplantation. Curr Opin Ophthalmol. 2003;14(6):433-442.

38. Maillet $S$, et al. Herpes simplex virus type 1 latently infected neurons differentially express latency-associated and ICP0 transcripts. J Virol. 2006;80(18):9310-9321.

39. Griffin DE. Viral encephalomyelitis. PLoS Pathog. 2011;7(3):e1002004.

40. Greene IP, Lee EY, Prow N, Ngwang B, Griffin DE. Protection from fatal viral encephalomyelitis: AMPA receptor antagonists have a direct effect on the inflammatory response to infection. Proc Natl Acad Sci U S A. 2008;105(9):3575-3580.

41. Bangham CR, Osame M. Cellular immune response to HTLV-1. Oncogene. 2005;24(39):6035-6046.

42. Matsuoka E, et al. Perivascular T cells are infected with HTLV-I in the spinal cord lesions with HTLV-Iassociated myelopathy/tropical spastic parapare- sis: double staining of immunohistochemistry and polymerase chain reaction in situ hybridization. Acta Neuropathol. 1998;96(4):340-346.

43. Jeffery KJ, et al. The influence of HLA class I alleles and heterozygosity on the outcome of human $\mathrm{T}$ cell lymphotropic virus type I infection. J Immunol. 2000;165(12):7278-7284.

44. Husby G, van de Rijn I, Zabriskie JB, Abdin ZH, Williams RC Jr. Antibodies reacting with cytoplasm of subthalamic and caudate nuclei neurons in chorea and acute rheumatic fever. J Exp Med. 1976;144(4):1094-1110.

45. Kirvan CA, Cox CJ, Swedo SE, Cunningham MW. Tubulin is a neuronal target of autoantibodies in Sydenham's chorea. J Immunol. 2007; 178(11):7412-7421

46. Kirvan CA, Swedo SE, Heuser JS, Cunningham MW. Mimicry and autoantibody-mediated neuronal cell signaling in Sydenham chorea. Nat Med. 2003;9(7):914-920.

47. Huerta PT, Kowal C, DeGiorgio LA, Volpe BT, Diamond B. Immunity and behavior: antibodies alter emotion. Proc Natl Acad Sci U S A. 2006; 103(3):678-683.

48. Tschen SI, Stohlman SA, Ramakrishna C, Hinton DR, Atkinson RD, Bergmann CC. CNS viral infection diverts homing of antibody-secreting cells from lymphoid organs to the CNS. EurJ Immunol. 2006;36(3):603-612.

49. Wakim LM, Woodward-Davis A, Bevan MJ. Memory $T$ cells persisting within the brain after local infection show functional adaptations to their tissue of residence. Proc Natl Acad Sci U S A. 2010;107(42):17872-17879.

50. Verjans GM, et al. Selective retention of herpes simplex virus-specific T cells in latently infected human trigeminal ganglia. Proc Natl Acad Sci US A. 2007;104(9):3496-3501.

51. Knickelbein JE, Khanna KM, Yee MB, Baty CJ, Kinchington PR, Hendricks RL. Noncytotoxic lytic granule-mediated CD8 $+\mathrm{T}$ cell inhibition of HSV-1 reactivation from neuronal latency. Science. 2008;322(5899):268-271.

52. Panzer J, Dalmau J. Movement disorders in paraneoplastic and autoimmune disease. Curr Opin Neurol. 2011;24(4):346-353.

53. Hughes EG, et al. Cellular and synaptic mechanisms of anti-NMDA receptor encephalitis. J Neurosci. 2010;30(17):5866-5875.

54. Tiwari JL, Terasaki PIE. HLA And Disease Associations. New York, New York, USA: Springer-Verlag; 1985:152-167.

55. Baranzini SE. The genetics of autoimmune diseases: a networked perspective. Curr Opin Immunol. 2009;21(6):596-605.

56. Bartholomaus I, et al. Effector $\mathrm{T}$ cell interactions with meningeal vascular structures in nascent autoimmune CNS lesions. Nature. 2009;462(7269):94-98.

57. Lovato L, et al. Related B cell clones populate the meninges and parenchyma of patients with multiple sclerosis. Brain. 2011;134(pt 2):534-541.

58. Ponsford M, Mazza G, Coad J, Campbell MJ, Zajicek J, Wraith DC. Differential responses of CD45+ve T-cell subsets to MBP in multiple sclerosis. Clin Exp Immunol. 2001;124(2):315-322.

59. Compston A, Coles A. Multiple sclerosis. Lancet. 2008;372(9648):1502-1517.

60. Jarius S, Wildemann B. AQP4 antibodies in neuromyelitis optica: diagnostic and pathogenetic relevance. Nat Rev Neurol. 2010;6(7):383-392.

61. Wingerchuk DM, Lennon VA, Pittock SJ, Lucchinetti CF, Weinshenker BG. Revised diagnostic criteria for neuromyelitis optica. Neurology. 2006;66(10):1485-1489.

62. Saadoun S, Waters P, Bell BA, Vincent A, Verkman AS, Papadopoulos MC. Intra-cerebral injection of neuromyelitis optica immunoglobulin G and human complement produces neuromyelitis opti- 
ca lesions in mice. Brain. 2010;133(pt 2):349-361.

63. Bradl M, et al. Neuromyelitis optica: pathogenicity of patient immunoglobulin in vivo. Ann Neurol. 2009;66(5):630-643.

64. Matsuya N, et al. Increased T-cell immunity against aquaporin-4 and proteolipid protein in neuromyelitis optica. Int Immunol. 2011;23(9):565-573.

65. Marrosu MG, et al. Multiple sclerosis in Sardinia is associated and in linkage disequilibrium with HLA-DR3 and -DR4 alleles. Am J Hum Genet. 1997; 61(2):454-457.

66. Compston A, Coles A. Multiple sclerosis. Lancet. 2002;359(9313):1221-1231.

67. Yamasaki K, et al. HLA-DPB1*0501-associated opticospinal multiple sclerosis: clinical, neuroimaging and immunogenetic studies. Brain. 1999;122(pt 9):1689-1696.

68. Zephir H, et al. Is neuromyelitis optica associated with human leukocyte antigen? Mult Scler. 2009;15(5):571-579.

69. Brum DG, et al. HLA-DRB association in neuromyelitis optica is different from that observed in multiple sclerosis. Mult Scler. 2010;16(1):21-29.

70. Fontana A, Gast H, Reith W, Recher M, Birchler T, Bassetti CL. Narcolepsy: autoimmunity, effector $\mathrm{T}$ cell activation due to infection, or $\mathrm{T}$ cell independent, major histocompatibility complex class II induced neuronal loss? Brain. 2010 133(pt 5):1300-1311

71. Mignot E, et al. Complex HLA-DR and -DQ interactions confer risk of narcolepsy-cataplexy in three ethnic groups. Am J Hum Genet. 2001;68(3):686-699.

72. Hallmayer J, et al. Narcolepsy is strongly associated with the T-cell receptor alpha locus. Nat Genet. 2009;41(6):708-711.

73. Hohjoh $\mathrm{H}$, et al. Significant association of a single nucleotide polymorphism in the tumor necrosis factor-alpha (TNF-alpha) gene promoter with human narcolepsy. Tissue Antigens. 1999;54(2):138-145.

74. Hohjoh $\mathrm{H}$, et al. Case-control study with narcoleptic patients and healthy controls who, like the patients, possess both HLA-DRB1*1501 and -DQB1*0602. Tissue Antigens. 2001;57(3):230-235.

75. Schuld A, Uhr M, Pollmacher T. Oligoclonal bands and specific antibody indices in human narcolepsy. Somnologie. 2004;8(3):71-74.

76. Aran A, et al. Elevated anti-streptococcal antibodies in patients with recent narcolepsy onset. Sleep. 2009;32(8):979-983.
77. Honda $\mathrm{M}$, et al. Absence of ubiquitinated inclusions in hypocretin neurons of patients with narcolepsy. Neurology. 2009;73(7):511-517.

78. Axtell RC, et al. T helper type 1 and 17 cells determine efficacy of interferon-beta in multiple sclerosis and experimental encephalomyelitis. Nat Med. 2010;16(4):406-412.

79. Coles AJ, et al. The window of therapeutic opportunity in multiple sclerosis: evidence from monoclonal antibody therapy. J Neurol. 2006;253(1):98-108.

80. Hauser SL, et al. B-cell depletion with rituximab in relapsing-remitting multiple sclerosis. NEnglJ Med. 2008;358(7):676-688

81. Clifford DB, De Luca A, Simpson DM, Arendt G, Giovannoni G, Nath A. Natalizumab-associated progressive multifocal leukoencephalopathy in patients with multiple sclerosis: lessons from 28 cases. Lancet Neurol. 2010;9(4):438-446.

82. Koralnik IJ. Progressive multifocal leukoencephalopathy revisited: Has the disease outgrown its name? Ann Neurol. 2006;60(2):162-173.

83. Cohen JA, et al. Oral fingolimod or intramuscular interferon for relapsing multiple sclerosis. $N$ Engl J Med. 2010;362(5):402-415.

84. Harrison LC, Hafler D. Antigen-specific therapy for autoimmune disease. Curr Opin Immunol. 2000;12(6):704-711.

85. Sloan-Lancaster J, Allen PM. Altered peptide ligand-induced partial T cell activation: molecular mechanisms and role in T cell biology. Annu Rev Immunol. 1996;14:1-27.

86. Teitelbaum D, Arnon R, Sela M. Copolymer 1: From basic research to clinical application. Cell Mol Life Sci. 1997;53(1):24-28.

87. Duda PW, Schmied MC, Cook SL, Krieger JI, Hafler DA. Glatiramer acetate (Copaxone) induces degenerate, Th2-polarized immune responses in patients with multiple sclerosis. J Clin Invest. 2000;105(7):967-976.

88. Tennakoon DK, Mehta RS, Ortega SB, Bhoj V, Racke MK, Karandikar NJ. Therapeutic induction of regulatory, cytotoxic CD8+ T cells in multiple sclerosis. J Immunol. 2006;176(11):7119-7129.

89. Kappos L, et al. Induction of a non-encephalitogenic type $2 \mathrm{~T}$ helper-cell autoimmune response in multiple sclerosis after administration of an altered peptide ligand in a placebo-controlled, randomized phase II trial. Nat Med. 2000;6(10):1176-1182.

90. Bielekova B, et al. Encephalitogenic potential of the myelin basic protein peptide (amino acids $83-99$ ) in multiple sclerosis: Results of a phase II clinical trial with an altered peptide ligand. Nat Med. 2000;6(10):1167-1175.

91. Anderton SM, et al. Fine specificity of the myelin-reactive $\mathrm{T}$ cell repertoire: implications for TCR antagonism in autoimmunity. J Immunol. 1998;161(7):3357-3364.

92. Warren KG, Catz I, Ferenczi LZ, Krantz MJ. Intravenous synthetic peptide MBP8298 delayed disease progression in an HLA Class II-defined cohort of patients with progressive multiple sclerosis: results of a 24-month double-blind placebo-controlled clinical trial and 5 years of follow-up treatment. Eur J Neurol. 2006;13(8):887-895.

93. Bar-Or A, et al. Induction of antigen-specific tolerance in multiple sclerosis after immunization with DNA encoding myelin basic protein in a randomized, placebo-controlled phase 1/2 trial. Arch Neurol. 2007;64(10):1407-1415.

94. Vernino S, et al. Paraneoplastic chorea associated with CRMP-5 neuronal antibody and lung carcinoma. Ann Neurol. 2002;51(5):625-630.

95. Dalmau J, Lancaster E, Martinez-Hernandez E, Rosenfeld MR, Balice-Gordon R. Clinical experience and laboratory investigations in patients with anti-NMDAR encephalitis. Lancet Neurol. 2011; 10(1):63-74.

96. Mathew RM, et al. Orchiectomy for suspected microscopic tumor in patients with anti-Ma2-associated encephalitis. Neurology. 2007;68(12):900-905.

97. Rojas I, et al. Long-term clinical outcome of paraneoplastic cerebellar degeneration and anti-Yo antibodies. Neurology. 2000;55(5):713-715.

98. Bernal F, et al. Anti-Tr antibodies as markers of paraneoplastic cerebellar degeneration and Hodgkin's disease. Neurology. 2003;60(2):230-234.

99. Graus F, Lang B, Pozo-Rosich P, Saiz A, Casamitjana R, Vincent A. P/Q type calcium-channel antibodies in paraneoplastic cerebellar degeneration with lung cancer. Neurology. 2002;59(5):764-766.

100 . Geis C, et al. Stiff person syndrome-associated autoantibodies to amphiphysin mediate reduced GABAergic inhibition. Brain. 2010;133(11):3166-3180.

101. Raju R, et al. Autoimmunity to GABAA-receptorassociated protein in stiff-person syndrome. Brain. 2006;129(Pt 12):3270-3276

102. Blaes F, Pike MG, Lang B. Autoantibodies in childhood opsoclonus-myoclonus syndrome. J Neuroimmunol. 2008;201-202:221-226. 